\title{
DEVELOPMENT OF THE IRON-CORED ELECTROMAGNET FOR THE TREATMENT OF NON-UNION AND DELAYED UNION
}

\author{
E. M. DOWNES, J. WATSON
}

From Singleton and Morriston Hospitals and the University of Wales, Swansea

\begin{abstract}
The development of the iron-cored electromagnet as a stimulator of bony union is described. In a clinical trial extending over eight years, 80 patients were treated for various forms of failed union. Their results are presented, as well as tentative conclusions regarding the indications and efficiency of the method. The latest, fully-portable apparatus is described.
\end{abstract}

The stimulation of healing in ununited fractures by the passage of electric currents is by no means new, for some allegedly successful treatments were reported during the nineteenth century. However, a seminal sequence of basic and animal experiments by Fukada and Yasuda (1957) in Japan, and by Bassett and Becker (1962) and Friedenburg and Brighton (1966) in the United States, led to the first successful clinical application, the healing of a case of non-union of the medial malleolus, which was reported by Friedenburg, Harlow and Brighton (1971).

The use of current-carrying electrodes has become well-known and some of the available commercial equipment has been reviewed elsewhere by one of the present authors (Watson 1983). Even now it is not clear whether the actual passage of current, the electrochemical processes at the cathode (where osteogenesis preferentially occurs), or a combination of both, is primarily responsible for the stimulation of healing.

In the absence of electrodes, however, it must be the passage of current which is responsible. Currents may readily be induced by the external application of pulsing magnetic fields. Such a field, oriented transversely across a limb, will induce a pulsing current within the limb. The induced current circulates around the axis of the magnetic field, as in Figure 1. It will rotate alternately one way, then the other, as the magnetic field rises and falls.

Two simple methods are available to produce appropriate magnetic fields. A pair of coils may be placed one on each side of the limb, connected in series, and then energised by a pulsing primary current (Fig. 2). If the diameters of the coils are greater than their distance apart, the magnetic field generated will be spatially uniform in the volume between them. Alternatively, a

E. M. Downes, FRCS, Consultant Orthopaedic Surgeon Singleton Hospital, Swansea, West Glamorgan, Wales.

J. Watson, PhD, FIEE, Reader in Electronic Engineering University of Wales, Swansea SA2 8PP, Wales.

Requests for reprints should be sent to Mr E. M. Downes.

(C) 1984 British Editorial Society of Bone and Joint Surgery $0301-620 \mathrm{X} / 84 / 5131 \$ 2.00$ non-uniform but more concentrated field may be produced by a single coil wound on an iron core (Fig. 3), when it is similarly energised. For either method, the primary current is derived from an electronic driver unit.

Each method results in a pulsing secondary current in the limb, but the varying resistances of tissues within the limb (bone has the highest resistance) cause an

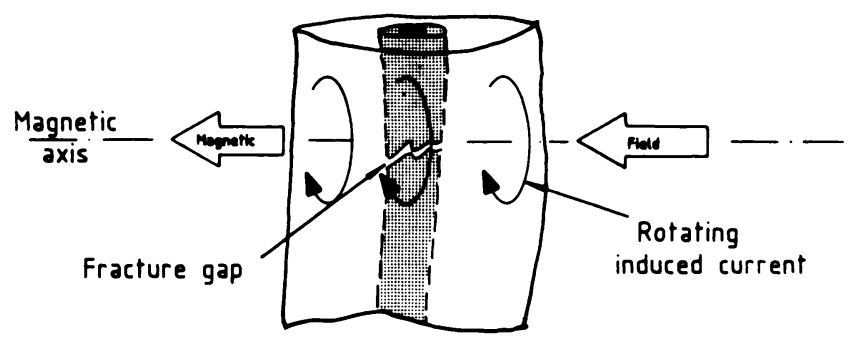

Fig. 1

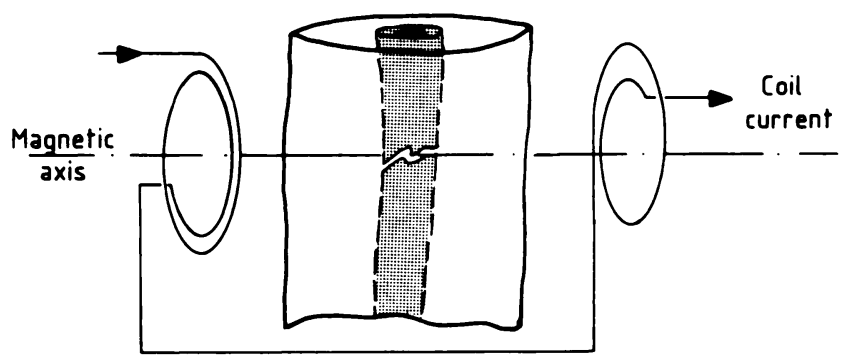

Fig. 2

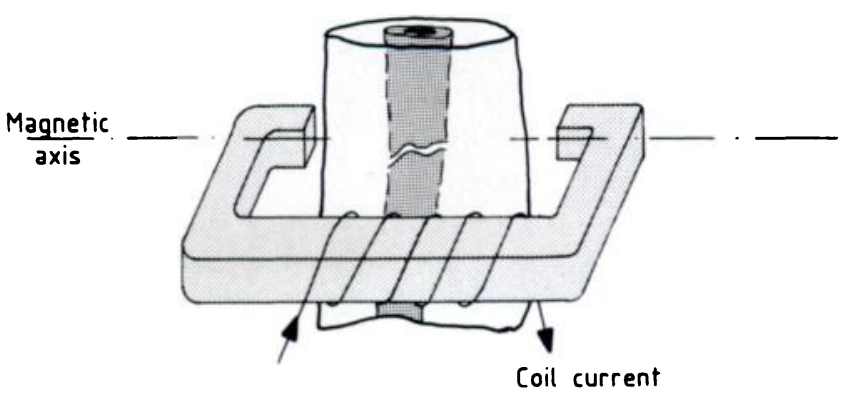

Fig. 3

The induction of current in a limb. Figure 1 -To show the induction of a rotating current. Figure 2-The use of series-connected Helmholz coils. Figure 3-The use of an electromagnet. 


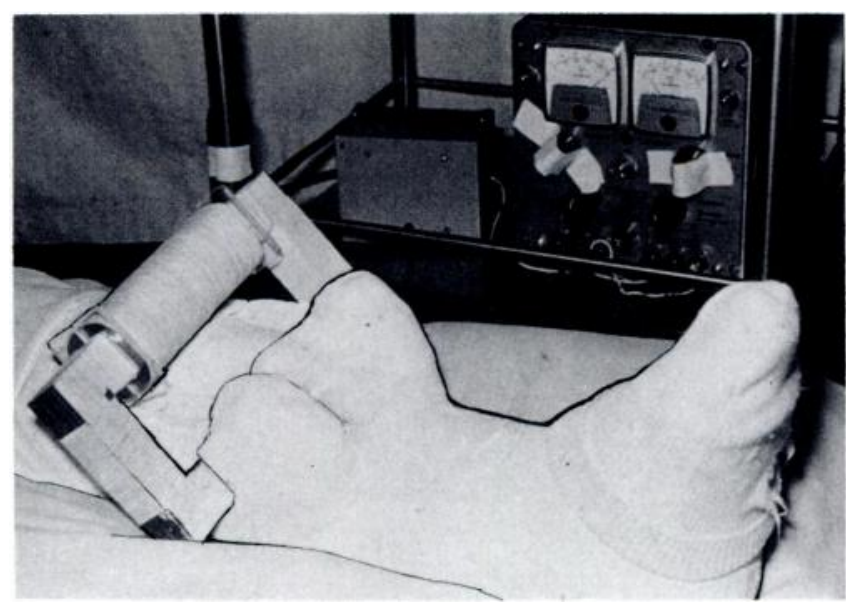

Fig. 4

The original heavy iron-cored electromagnet in use in 1974.

extremely complex current density distribution and pattern of associated potential differences (Lunt 1982).

Air-cored coils were used by Bassett, Pawluk and Pilla (1974) to apply pulsed magnetic fields across fibular osteotomies in dogs and this work was followed by a series of clinical trials (Bassett, Mitchell and Gaston 1982) culminating in the commercial development of apparatus for bone stimulation. An iron-cored electromagnet was successfully used by de Haas and Watson (1975) in the treatment of a fractured tibia still un-united after five years. This electromagnet, which contained laminations from a power transformer, weighed a formidable $7 \mathrm{~kg}$ with its winding (Fig. 4). A clinical trial with this device was reported by de Haas, Watson and Morrison (1980).

A new orthopaedic stimulator, designed and built at Swansea in the mid 1970s, and subsequently modified, has been used clinically for eight years. This apparatus and its use form the basis of this present paper.

\section{MATERIALS AND METHODS}

The original Swansea heavy orthopaedic stimulator electromagnet (designated OSH) was built up to a $25 \mathrm{~mm}$ square cross-section from laminated strips of Telcon Metals grain-oriented steel, and when wound it weighed $2.9 \mathrm{~kg}$. The coil was energised by a symmetrical square voltage wave as shown in Figure 5a with an amplitude of 38 volts and a frequency of about $1 \mathrm{~Hz}$. This frequency was chosen arbitrarily within limits imposed by the inductance of the system, and because it roughly corresponded to such human biological frequencies as heart rate and normal walking rhythm. Although the voltage wave applied to the coil was square, the resulting primary current waveform rose and fell exponentially as a result of the high inductance, as seen in Figure $5 \mathrm{~b}$. The magnetic field rose and fell with this primary current, and resulted in an induced secondary current having the waveform shown in Figure 5c. This waveform was obtained directly by using a small search coil consisting of 50 turns of $24 \mathrm{~s}$.w.g. wire on a $5 \mathrm{~mm}$ diameter former.
This coil was held in contact with one of the pole faces and the induced voltage was recorded on an oscilloscope. This shows clearly that the magnitude of such an induced voltage - and hence of the secondary current in the limb-varies with the rate of change of the primary current and therefore with the magnetic field.

The peak value of this waveform was $2.5 \mathrm{mV}$, and the mid-pole field strength produced was about 120 gauss (compared with 150 gauss for the original Canadian magnet). This magnet was applied clinically using wooden guides placed on either side of the plaster cast at the level of the non-union, ensuring accuracy by control radiographs and metal markers. In this way the poles of the magnet could, during treatment periods, be placed so that the axis of the magnetic field was at right angles to the axis of the bone and across the site of non-union. Removal and replacement of the magnet could easily be done by the patient.

The first four patients in the series were treated in hospital to allow observation and to facilitate correction of any problems which might arise during treatment. No difficulties were in fact encountered; since then almost all patients have been treated at home. For the earlier patients treatment was given for 20 hours per day for six weeks, with continued plaster splintage for another six weeks, after which clinical and radiological assessment was undertaken. In cases involving the lower limb, weight-bearing was not permitted. If union was not

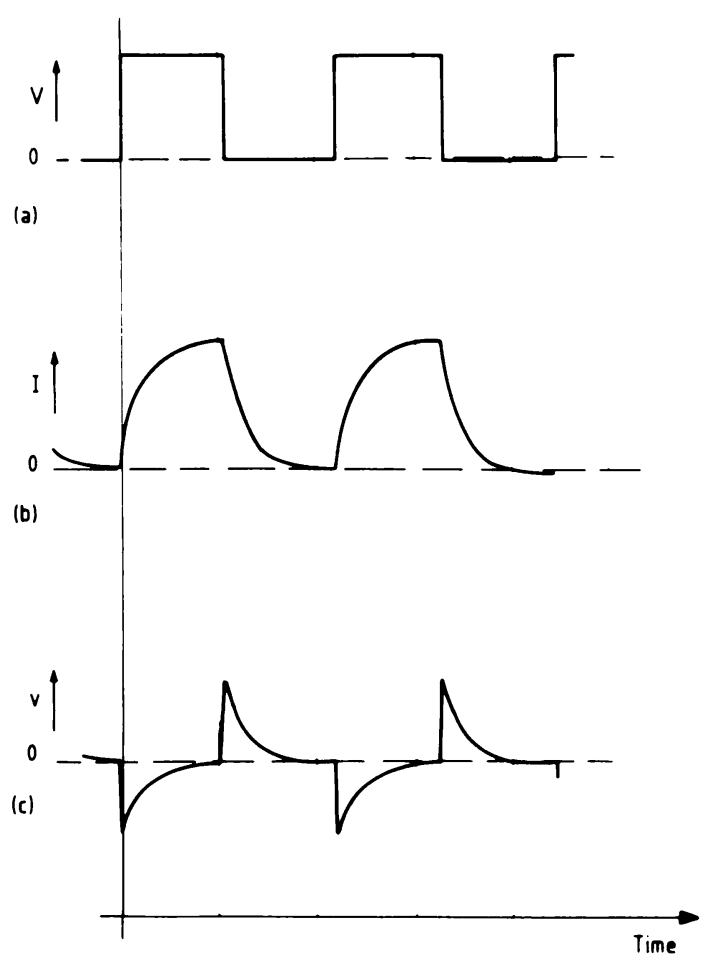

Fig. 5

Diagram to show the waveforms of voltage $(a)$ and current (b) in the original electromagnet. The voltage induced in the search coil placed at a pole face is at $(c)$. The time scale divisions are at 0.5 second intervals. 
evident after 12 weeks, plaster was retained, with further assessment each month for three months.

Over a three-year period, 31 patients with various types of delayed and non-union were treated by this regime and are designated Group 1. Two patients were withdrawn from this group because of incomplete treatment caused by lack of co-operation or failure of equipment.

Concern that the heavy magnet and the long immobilisation during treatment were reducing the acceptability of the treatment, and thus the co-operation of the patients, led to further development in 1979. The core thickness of the magnet was reduced to $18 \mathrm{~mm}$, the peak mid-pole flux density to about 75 gauss and the weight to $1.8 \mathrm{~kg}$. Frequency stayed at $1 \mathrm{~Hz}$. This new core, OSL (A) (Fig. 6), produced a peak induced voltage

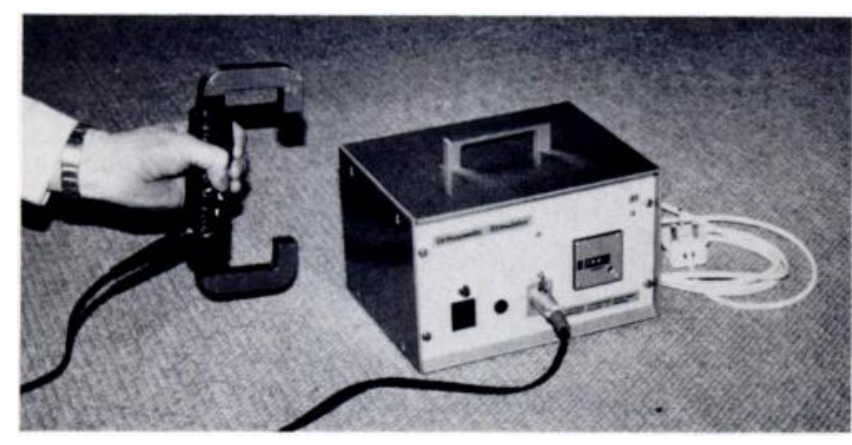

Fig. 6.

The developed OSL (A) electromagnet and electronic driver.

at the pole face of $1.8 \mathrm{mV}$ measured with the same search coil. The daily period of treatment was reduced to 12 hours and continued for 12 weeks. Assessment for union was as before. Over two years 18 patients with 19 nonunions were treated with this new magnet, and these constitute Group 2.

At this stage it was felt that a higher induced voltage was indicated, so the winding on the same core and the drive were redesigned to produce a peak induced voltage at the pole face of $3.5 \mathrm{mV}$. The frequency was increased to seven induced pulses per second, this being possible because of the lower inductance of the new electromagnet. Because the induced voltage is proportional to the rate of change of field strength (and not to its absolute magnitude), the mid-pole field strength was reduced to only 36 gauss. This unit was designated OSL (B), and was used over the next two years to treat 33 patients for 12 hours a day over 12 weeks as was done with the OSL (A) unit. These patients are designated Group 3.

The developments so far described stemmed from the heavy empirical designs produced in Canada before specifications could be formulated. However, continued experience, fundamental studies such as those of Pilla (1980) and, especially, concern for the convenience of patients and acceptability, led to the production of a completely new system.

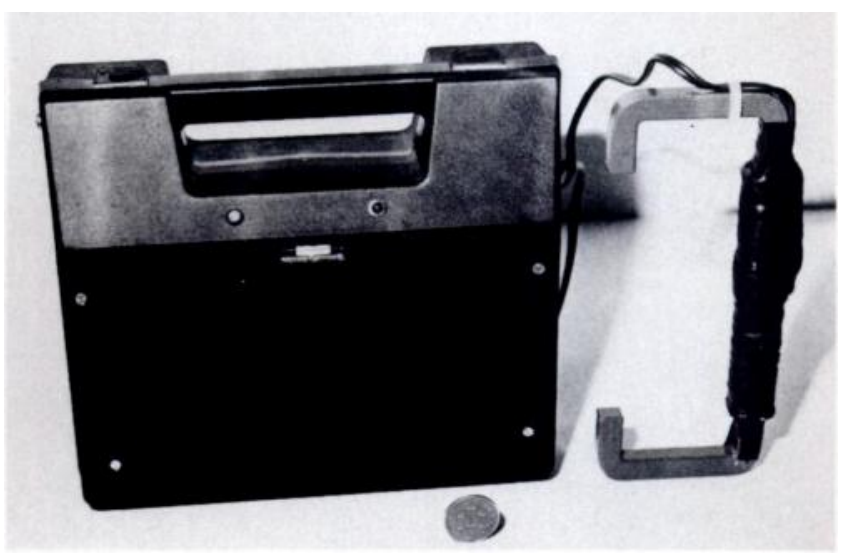

Fig. 7

The new light-weight fully portable electromagnet and its battery powered drive unit.

This uses an electromagnet of $10 \mathrm{~mm}$ square crosssection, weighing only $0.5 \mathrm{~kg}$, which may be incorporated into a plaster cast in the appropriate position. Its coil is driven from a fully portable electronic unit weighing only $1.5 \mathrm{~kg}$ inclusive of a $12 \mathrm{~V}$ rechargeable battery (Fig. 7). In the interests of battery conservation, the electronic drive system and electromagnet have been completely redesigned, and result in the wave shapes shown in Figure 8. A rectangular $12 \mathrm{~V}, 10 \mathrm{~ms}$ voltage pulse is applied to the low-resistance, low-inductance electromagnet coil at a frequency of 10 to 15 pulses per second (Fig. $8 \mathrm{a})$. This results in a current waveshape shown in Figure $8 \mathrm{~b}$ where the fast rise is followed by a slow, exponential fall. The voltage induced in the search coil has the form of Figure 8c, which is markedly asymmetrical.

The treatment programme has also been changed, as the portability of the unit has allowed return to 20 hours per day treatment time, while permitting the patient to remain mobile and, where practicable, to work. Four patients have been treated, three successfully, using this technique; the first of these has been briefly reported elsewhere (Watson and Downes 1983).

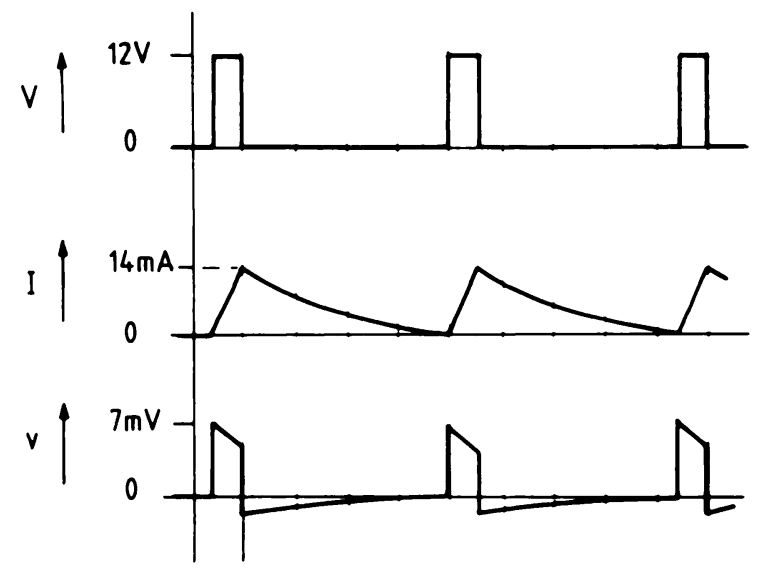

(a)

Fig. 8

Diagram to show the waveforms of voltage ( $a$ ) and current $(b)$ applied to the new light-weight electromagnet. The voltage induced in the search coil at the pole face is at $(c)$. The time scale divisions are at $20 \mathrm{~ms}$ intervals. 


\section{RESULTS}

The results from 81 failed unions in 80 patients are reported. Four patients were excluded from the series, three because they failed to complete the course of treatment and the fourth because he had an operation as well as magnetic stimulation. The criterion of success was the clinical and radiological demonstration of bony union after a course of electromagnetic stimulation with no additional treatment other than plaster splintage.

Over the eight years of this study, various changes in treatment were made, both in electrical values and in the duration of stimulation, with the aims of determining the factors which influence the success rate, and of improving patient acceptability.

The three groups of patients, treated respectively with the OSH, the OSL (A) and the OSL (B) equipment, providing the treatment levels and durations given above, had results as follows: Group 1, 29 patients, had a $69 \%$ success rate; Group 2, 18 patients with 19 failed unions, had a $63 \%$ success rate; while Group 3, 33 patients, had a $70 \%$ success rate.

It was evident that no significant change in success rate had followed the changes in treatment. For this reason, and to provide larger numbers, all three groups were used to assess the influence of other factors on the chances of success. The grouped results were considered in terms of the age and sex of the patient, the site of failed union, its duration, and the nature of previous treatment.

Table I shows the number of successes related to age. Although the numbers are too small for statistical analysis, the impression is that success is greatest in the youngest and (surprisingly) the oldest age groups. No difference in results by sex was shown-of 57 male patients 40 united, while 16 of 24 female patients had a successful result.

The sites of non-union given in Table II reflect the well-known preponderance of non-union in the tibia. Allowing for the small numbers, success with the proximal long bones, the femur and the humerus, is surprisingly high considering the difficulty of immobilising these fractures in plaster. The duration of failed union before treatment is shown in Table III. The success rate for cases of delayed union (from four months to nine months after fracture) was $73 \%$, as against $65 \%$ for cases of 10 months and over (regarded as having established non-union). If, however, cases of more than two years' duration are excluded, the success rate for the cases from 10 to 23 months is $79 \%$. In this series, when the duration of the non-union was more than 24 months the chances of achieving success using electrical stimulation alone was poor; only 4 of 12 such patients obtained union. This contrasts with Sharrard's series in which the results were unaffected by the time which had elapsed between the accident and the start of electrical treatment (Sharrard et al. 1982).

More than half of our patients had been previously
Table I. Age of patients related to success of treatment

\begin{tabular}{|c|c|c|c|c|c|}
\hline & \multicolumn{5}{|c|}{ Age (years) } \\
\hline & $<21$ & 21-35 & $36-50$ & $51-64$ & $65+$ \\
\hline Cases & 15 & 32 & 10 & 15 & 9 \\
\hline Union & 15 & 21 & 5 & 8 & 8 \\
\hline$\%$ success & 100 & 65 & 50 & 53 & 89 \\
\hline
\end{tabular}

Table II. Site of non-union related to success of treatment

\begin{tabular}{|llllllll|}
\hline \multicolumn{4}{ll}{ Site of non-union } \\
\cline { 2 - 7 } & Tibia & Femur & Humerus & Ulna & Clavicle & $\begin{array}{l}\text { Knee } \\
\text { arthrodesis Ankle }\end{array}$ \\
\hline Cases & 55 & 8 & 5 & 3 & 2 & 5 & 2 \\
Union & 37 & 7 & 4 & 2 & 1 & 3 & 2 \\
\hline
\end{tabular}

Table III. Duration of delayed and non-union before treatment related to the success of treatment

\begin{tabular}{|c|c|c|c|c|c|}
\hline & \multicolumn{5}{|c|}{ Duration of delayed and non-union (months) } \\
\hline & $4-6$ & $7-9$ & 10-12 & $13-23$ & $24+$ \\
\hline Cases & 24 & 17 & 13 & 15 & 12 \\
\hline Union & 18 & 12 & 9 & 13 & 4 \\
\hline$\%$ success & & & & & 33 \\
\hline
\end{tabular}

Table IV. Previous treatment related to the success of electrical treatment

\begin{tabular}{|llllc|}
\hline & \multicolumn{4}{l}{ Operations } \\
\cline { 3 - 5 } & Conservative & 1 & 2 & $3+$ \\
\hline Cases & 45 & 17 & 15 & 4 \\
Union & 35 & 9 & 10 & 2 \\
$\%$ success & 78 & 53 & 67 & 50 \\
\hline
\end{tabular}

treated by conservative means only, most by plaster; the others had had one or more operations. The group previously treated conservatively had a better success rate with electrical treatment than those who had had operations, but there was no correlation between the number of operations and the success rate, the relationship being shown in Table IV.

\section{DISCUSSION}

Failure of union nine months after fracture was adopted as the criterion of non-union (Müller, Allgöwer and Willeneger 1965). Treatment was also given to some fractures un-united after a shorter period (never less than four months) on the basis that the referring surgeon would otherwise have undertaken operative treatment.

To avoid confusing the results, patients receiving any treatment other than electrical stimulation in a 


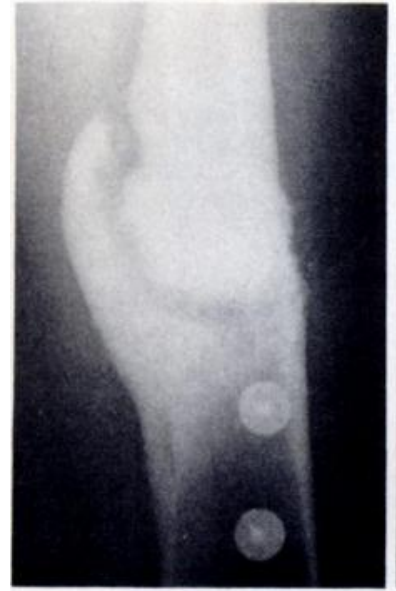

Fig. 9

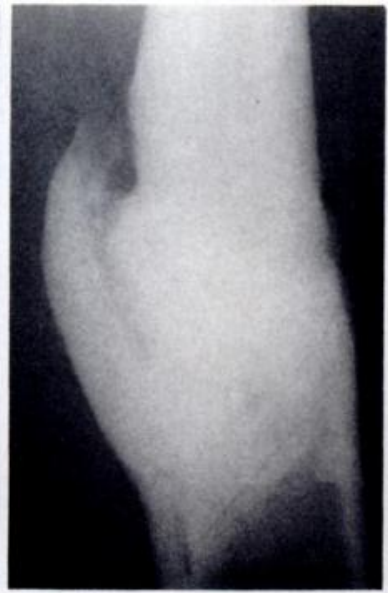

Fig. 10

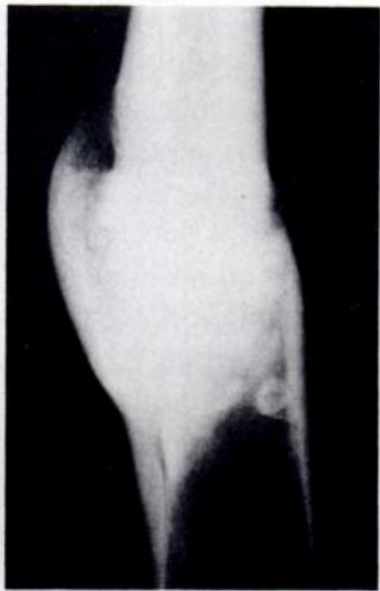

Fig. 11

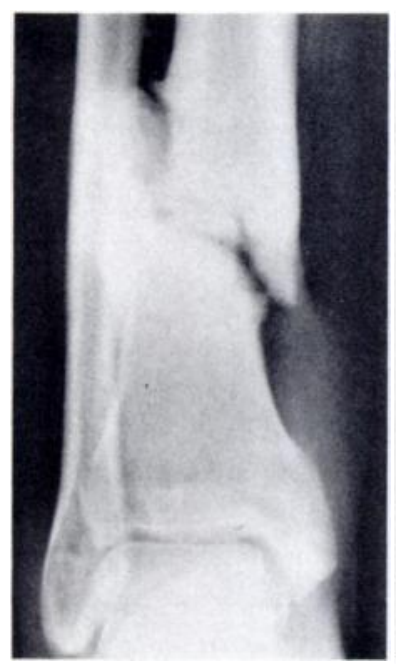

Fig. 12

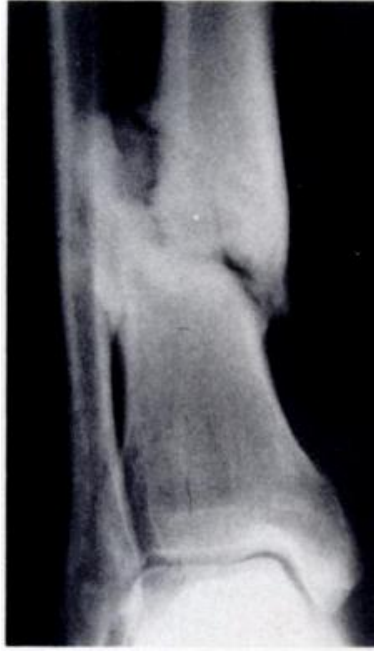

Fig. 13

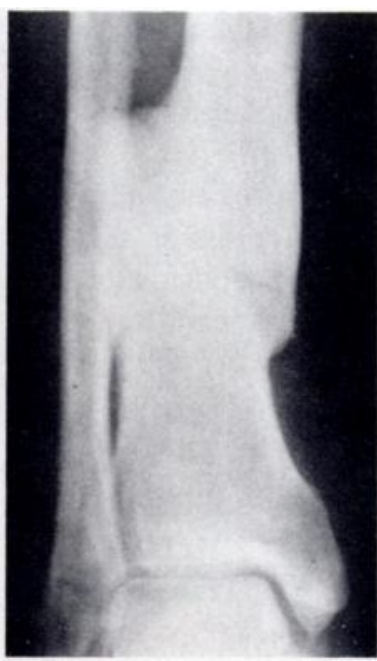

Fig. 14
Radiographs of a patient with non-union of the femur, 22 months after injury and an unsuccessful bone graft. Figure 9-Before treatment, to show markers used for positioning. Figure 10-After 12 weeks' treatment showing clouding of the fracture line. Figure 11-Consolidation 26 weeks after starting treatment.
Radiographs of the lower end of the right tibia. Figure 12-Un-united fracture 18 months after the original injury. Figure 13-There is some clouding of the fracture gap after 12 weeks. Figure 14-The fracture consolidated 10 months after starting electrical treatment. (Reproduced from Watson and Downes 1978, by kind permission of the Japanese Journal of Applied Physics.)

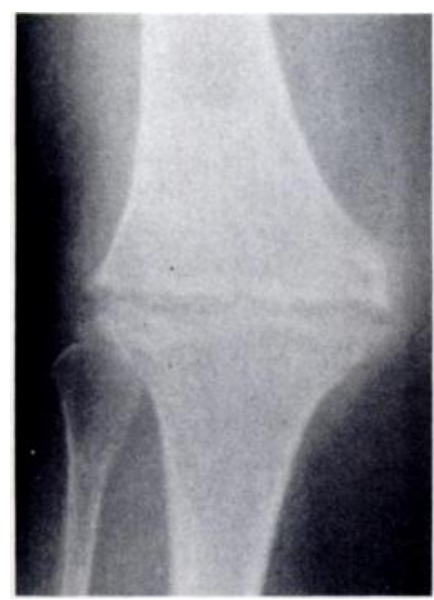

Fig. 15

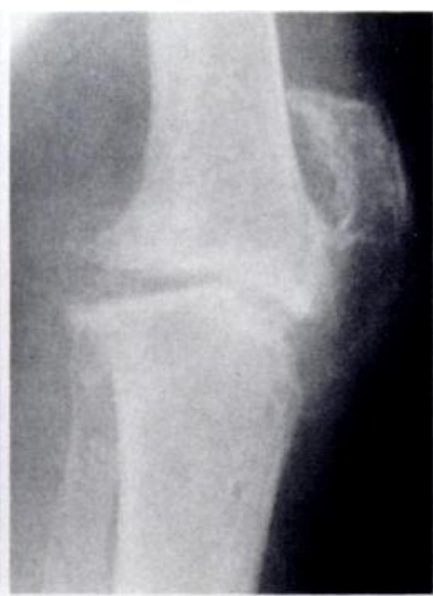

Fig. 16

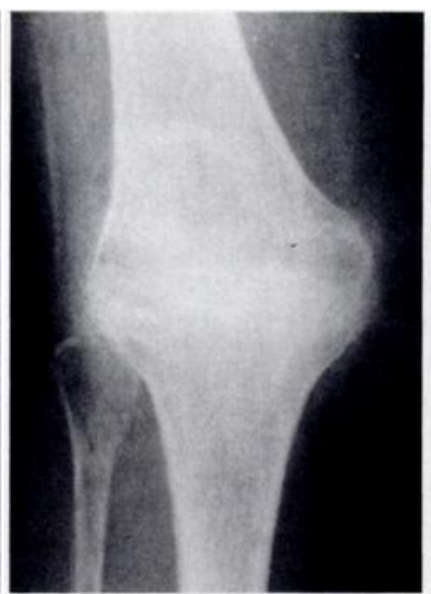

Fig. 17

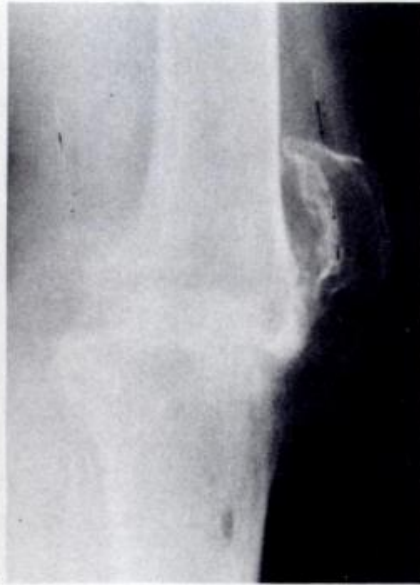

Fig. 18

Radiographs taken after an attempted arthrodesis of the right knee. Figures 15 and 16-Anteroposterior and lateral views 10 months after operation showing established non-union. Figures 17 and 18-Union is present after five months of electromagnetic treatment. 
plaster cast have been excluded; and as stated, success was defined as sound bony union demonstrable both clinically and radiologically.

It is of interest that the various changes of technique and timing made during the study failed to produce any significant change in the results. This may mean either that there is a simple "threshold effect" which promotes osteogenesis in successful cases, or that a more appropriate combination of parameters of treatment awaits discovery. The solution remains conjectural.

The radiographs of three typical cases are shown in Figures 9 to 18; these illustrate the radiological changes during the progress to union, with bony infilling, and virtual absence of external callus.

Ten patients all with established non-union of the tibia, had active infection with discharging sinuses. All had had bone grafts previously, some after internal fixation. Successful union was achieved in five of these difficult cases. During treatment they were carefully observed for increase in local sepsis. No harmful effects were seen; in fact there was actual reduction in local inflammation and discharge from sinuses, even when union was not achieved. In three of the patients gaining union the sinuses healed completely.

Three factors favoured success in our series. First, the youth of the patient: those aged 20 years or less did well. Although a high success rate was also achieved in patients aged over 65 , the small numbers involved preclude firm correlation. Secondly, an interval of under two years from the original injury gave better results than a longer interval. We found no difference in results between delayed union (under nine months) and nonunion (nine months to two years). Thirdly, previous conservative rather than operative management for the failed union favoured success.

Conclusions. The $70 \%$ success rate in this study shows that non-invasive magnetic stimulation is a feasible alternative to established surgical methods in treating un-united fractures; it seems reasonable to suppose that this applies also to failed osteotomies and arthrodeses.

The method is particularly applicable at five to six months after injury, when an orthopaedic surgeon may decide that union of a fracture is unlikely with splintage alone, and that additional treatment is needed. Potentially hazardous surgery may be avoided in a majority of such cases by the use of magnetic stimulation.

\section{REFERENCES}

Bassett CAL, Becker RO. Generation of electrical potentials by bone in response to mechanical stress. Science 1962;137:1063-4.

Bassett CAL, Pawluk RJ, Pilla AA. Acceleration of fracture repair by electromagnetic fields: a surgically noninvasive method. Ann NY Acad Sci $1974 ; 238: 242-62$.

Bassett CAL, Mitchell SN, Gaston SR. Pulsing electromagnetic field treatment in ununited fractures and failed arthrodeses. JAMA 1982;247: 623-8.

de Haas WG, Watson J. The noninvasive treatment of tibial nonunion using a pulsed magnetic field. Paper delivered at SICOT XIII World Congress, Copenhagen, 1975.

de Haas WG, Watson J, Morrison DM. Non-invasive treatment of ununited fractures of the tibia using electrical stimulation. $J$ Bone Joint Surg $[B r] 1980 ; 62-B: 465-70$.

Friedenburg ZB, Brighton CT. Bioelectric potentials in bone. J Bone Joint Surg [Am] 1966;48-A:915-23.

Friedenburg ZB, Harlow MC, Brighton CT. Healing of nonunion of the medial malleolus by means of direct current: a case report. $J$ Trauma 1971;11:883-5.

Fukada E, Yasuda I. On the peizoelectric effect of bone. J Phys Soc Japan 1957;12:1158-62.

Lunt MJ. Magnetic and electric fields produced during pulsed-magnetic-field therapy for non-union of the tibia. Med Biol Eng Comput 1982; $20(4): 501-11$.

Mäller ME, Allgöwer H, Willeneger H. Technique of internal fixation of fractures. Berlin : Springer-Verlag, 1965.

Pilla AA. Electrical modulation of biological regulatory processes via electrochemistry at cell surfaces. Paper delivered at the Chemical Society Electrochemistry Group Meeting, Oxford July 1-2, 1980.

Sharrard WJW, Sutclifie ML, Robson MJ, MacEachern AG. The treatment of fibrous non-union of fractures by pulsing electromagnetic stimulation. J Bone Joint Surg [Br] 1982;64-B: 189-93.

Watson J. The electrical stimulation of bone healing in cases of nonunion and delayed union. In: McKibbin B, ed. Recent advances in orthopaedics 4. Edinburgh, London, Melbourne, New York: Churchill Livingstone, 1983: 131-53.

Watson J, Downes EM. Application of pulsed magnetic fields to the stimulation of bone healing in humans. Jpn J Appl Phys 1978;17:215-7.

Watson J, Downes EM. Light-weight battery-operable orthopaedic stimulator for the treatment of long-bone nonunions using pulsed magnetic fields. Med Biol Eng Comput 1983;21 (4):509-10. 